\section{Supraoptimal Root-zone Temperatures Affect Dahlia Growth and Development}

\author{
Karen K. Schneck ${ }^{1}$, Cheryl R. Boyer ${ }^{1}$, and Chad T. Miller ${ }^{1}$
}

AdDITIONAL INDEX WORDs. controlled environment, dahlia decline, Dablia $\times$ bybrida, floriculture, greenhouse production, vegetative production

Summary. Dahlia (Dablia $\times$ bybrida) is an important floriculture crop that has gained popularity in recent years. Greenhouse growers have recently reported a phenomenon known as "dahlia decline," that can affect potted dahlias in greenhouse production. The crop exhibits graying foliage, root decline, and plant death, and the phenomenon has reportedly caused partial or total crop loss and has no known initiating factor. We hypothesized that plant exposure to supraoptimal root-zone temperatures (RZTs) during production may decrease dahlia root quality, especially above $40^{\circ} \mathrm{C}$ and could initiate dahlia decline. Because there is a lack of understanding on how supraoptimal RZT may impact dahlia growth and development, experiments were conducted to evaluate the effects of supraoptimal RZTs on seven dahlia cultivars in Spring 2019 and 2020. Dahlias were grown for 4 to 5 weeks in the greenhouse and then root zones were exposed to $\approx 22$ (control), $35,40,45$, or $50^{\circ} \mathrm{C}$ using a water bath. Root quality was rated before treatment and rated weekly after the hot water bath treatment, along with vegetative growth parameters for 4 weeks. In both years, significant decline in root ratings were observed. 'XXL Veracruz' and 'XXL Sunset' average root ratings decreased after a 45 and $50{ }^{\circ} \mathrm{C}$ treatments in year 2 and both cultivars demonstrated increased root rating averages by 3 weeks after treatment. Cultivars exhibited a significant increase in root rating in the final observations when compared with root ratings taken 1 week posttreatment even if the initial decline after treatment was not significant. Overall plant height was significantly impacted, resulting in shorter heights in both years for all cultivars as treatment temperatures increased to $50{ }^{\circ} \mathrm{C}$ in comparison with the control and $35^{\circ} \mathrm{C}$, and a few cultivars exhibited significantly shorter height at 40 and $45^{\circ} \mathrm{C}$. Ultimately, our research did not show typical plant responses that were consistent with reported dahlia decline, but we were better able to characterize dahlia response to supraoptimal RZT.

$\mathrm{D}$ ahlia (Dablia $\times$ bybrida) is a common annual garden plant that provides a variety of color

Received for publication 25 May 2021. Accepted for publication 29 July 2021.

Published online 24 September 2021

${ }^{1}$ Department of Horticulture and Natural Resources, Kansas State University, Manhattan, KS 66506

This manuscript has been assigned contribution no. 21-146-J from the Kansas Agricultural Experiment Station (KAES). This project was supported by the Fred C. Gloeckner Foundation. Its contents are solely the responsibility of the authors and do not necessarily represent the official views of the KAES or the Gloeckner Foundation. We thank Bob Van Sprange from Bosgraaf Greenhouses and Alicain Carlson from Syngenta Flowers, as well as greenhouse managers Lea Westervelt and Jacob Hueste, Jacob Schwab, and Bailee Davis from Kansas State University for assistance with this research. This article is from a thesis submitted by K.K. Schneck in partial fulfillment of the requirements for the M.S. degree.

K.K.S. is a Graduate Research Assistant.

C.R.B. and C.T.M. are Associate Professors.

C.T.M. is the corresponding author. E-mail: ctmiller@ksu.edu.

This is an open access article distributed under the CC BY-NC-ND license (https://creativecommons. org/licenses/by-nc-nd/4.0/).

https://doi.org/10.21273/HORTTECH04896-21 and interest to the landscape and is an important part of the modern floriculture and landscape industries. In the past several years, potted annual dahlias have gained popularity due to their versatility in planters and landscape plantings (Dolce, 2020; Schoellhorn, 2015). Dahlia was named Plant of the Year in 2019 by the National Garden Bureau (2019), further highlighting public interest in this species. Increased production of vegetatively propagated dahlia for use as annual landscape plants and novel cultivar development has led growers to a new and challenging problem: dahlia decline. Dahlia decline occurs during dahlia production near market date and has been reported in different dahlia series and breeding lines. Reported dahlia decline symptoms include plants exhibiting reduced rooting quality, graying foliage, foliage wilting, and plant death. Growers have indicated that plant symptoms affect significant numbers of plants (i.e., whole benches of crops) and near market stage in production (P.A. Hammer, personal communication). Dahlia decline thus far, to our knowledge, has only been reported during greenhouse production and associated with plants produced from vegetative cuttings. To date, no definitive cause or causes have been identified. One hypothesized explanation for dahlia decline is root damage induced by supraoptimal RZTs (P.A. Hammer, personal communication). When plant root zones are exposed to extreme ranges outside normal growing conditions, roots can be damaged, which can alter nutrient and water uptake, and ultimately affect plant growth and development, and in extreme cases, can result in plant death (Dodd et al., 2000; Ingram et al., 2015; Nambuthiri et al., 2015).

Other studies on supraoptimal RZTs have been reported in nursery crops such as foster holly (Ilex $\times$ attenuata) and lacebark elm (Ulmus parvifolia) (Martin et al., 1989; Yeager et al., 1991), southern magnolia [Magnolia grandiflora (Martin et al., 1991)], and vegetable crops including cucumber [Cucumis sativus (Du and Tachibana, 1994)], pepper [Capsicum annuum (Dodd et al., 2000)], potato (Solanum tuberosum), sweetpotato (Ipomoea batatas), and cassava (Manihot esculenta) (Sattelmacher et al., 1990). Studies on supraoptimal RZT effects on potted floriculture crops are limited. Information on suboptimal RZT modification for floriculture crop growth has been published on snapdragon [Antirrbinum majus (Hood and Mills, 1994; Wai and Newman, 1992)] and poinsettia [Euphorbia pulcherrima (Olberg and Lopez, 2016)]. However, these studies did not cover the supraoptimal RZT aspect of plant growth. Hood and Mills (1994) found that as RZTs increased, snapdragons improved their nutrient uptake and

\begin{tabular}{llll}
\hline $\begin{array}{l}\text { Units } \\
\text { To convert U.S. to SI, } \\
\text { multiply by }\end{array}$ & U.S. unit & SI unit & $\begin{array}{l}\text { To convert SI to U.S., } \\
\text { multiply by }\end{array}$ \\
\hline 2.54 & inch $(\mathrm{es})$ & $\mathrm{cm}$ & 0.3937 \\
1 & $\mathrm{ppm}$ & $\mathrm{mg} \cdot \mathrm{L}^{-1}$ & 1 \\
$\left({ }^{\circ} \mathrm{F}-32\right) \div 1.8$ & ${ }^{\circ} \mathrm{F}$ & ${ }^{\circ} \mathrm{C}$ & $\left({ }^{\circ} \mathrm{C} \times 1.8\right)+32$
\end{tabular}


Table 1. Dahlia series and cultivars used in experiments to further characterize dahlia decline phenomenon. Research was conducted in glass houses at Manhattan, KS.

\begin{tabular}{llcll}
\hline Series & \multicolumn{1}{c}{ Cultivar } & Group no. & Date liners received & Plant pinch date \\
\hline Dalaya & Red and White & 1 & 6 Feb. 2019;2 Feb. 2020 & 27 Feb. 2019; 20 Feb. 2020 \\
Dalaya & Shiva & 1 & 6 Feb. 2019;2 Feb. 2020 & 27 Feb. 2019; 20 Feb. 2020 \\
Dalaya & Yogi & 1 & 6 Feb. 2019;2 Feb. 2020 & 27 Feb. 2019; 20 Feb. 2020 \\
XXL & Veracruz & 2 & 22 Feb. 2019; 20 Feb. 2020 & 27 Feb. 2019; 20 Feb. 2020 \\
XXL & Tabasco & 2 & 22 Feb. 2019; 20 Feb. 2020 & 5 Mar. 2019; 20 Feb. 2020 \\
XXL & Sunset & 2 & 22 Feb. 2019; 20 Feb. 2020 & 5 Mar. 2019; 20 Feb. 2020 \\
Melody & Sincerity & 3 & 21 Mar. 2019; 20 Mar. 2020 & 1 Apr. 2019; 4 Apr. 2020 \\
\hline
\end{tabular}

growth. Wai and Newman (1992) reported that time to flower for cut flower snapdragon was reduced when RZTs increased even when air temperatures were cool. Although neither of these snapdragon studies discussed supraoptimal RZTs, they demonstrated the impact of RZT on growth and development of floriculture crops.

In an effort to characterize and understand the potential mechanisms involved in dahlia decline, we investigated supraoptimal RZT effects on several commercially available dahlia cultivars across different hybridized dahlia series over 2 years (2019 and 2020).

\section{Materials and methods}

Several dahlia cultivars from three dahlia series were used for this research (Table 1). Dahlias were received from commercial suppliers based on availability, which resulted in the formation of three experimental groups each year (Table 1). On receipt, rooted liners were immediately transplanted into 4.5-inch azalea plastic pots filled with a peat-based substrate (Sungro 3B Mix; Sun Gro Horticulture, Agawam, MA). In 2020, 'Melody Sincerity' plants were received as unrooted cuttings and were stuck in peat-based medium and rooted under frequent mist for $4 \mathrm{~d}$ then occasional mist for 2 weeks; after which, they were transferred to the greenhouse and further rooted for 3 weeks before they were transplanted.

Plants were grown in a glass greenhouse with temperature set points of $21 / 18^{\circ} \mathrm{C}$ (day/night) controlled by an environmental computer system (Titan; Argus Control Systems, Surrey, BC, Canada). High-pressure sodium lights (400 W, Lumalux S51; Sylvania, Danvers, MA) were used to provide a 6-h growing day extension, creating a 12-h total daylength. Fertilizer-injected municipal water $(\mathrm{pH}$ of $\approx 9$ and alkalinity of $\approx 40 \mathrm{ppm}$ ) and $\approx 200 \mathrm{ppm}$ nitrogen from $20 \mathrm{~N}-4.3 \mathrm{P}-16.6 \mathrm{~K}$ fertilizer (JR Peters, Allentown, PA) was used in this study. Media moisture levels were maintained at moist levels and fertigated when growing medium became dry but before plants began to wilt (Syngenta Flowers, 2019). This averaged out to every other day to daily as plant biomass increased. Plain water was used once per week. Pests were managed as needed and followed typical greenhouse production protocols. This included weekly applications of sodium bicarbonate (MilStop; BioWorks, Victor, NY) to manage powdery mildew (Erysiphe cichoracearum), and applications of spinosad (Conserve; Dow AgroSciences, Indianapolis, IN) to control thrips (Thysanoptera). Pesticide applications were made across all treatments of the entire dahlia crop. Biological fungicide applications (RootShield; BioWorks) were applied between 1 and 2 weeks after potting to reduce the incidence of root rot pathogens.

Dahlias were pinched 2 weeks after potting to leave two nodes, then spaced $\approx 20 \mathrm{~cm}$ on center on a metal bench. After 4 or 5 weeks of growth, depending on the root development

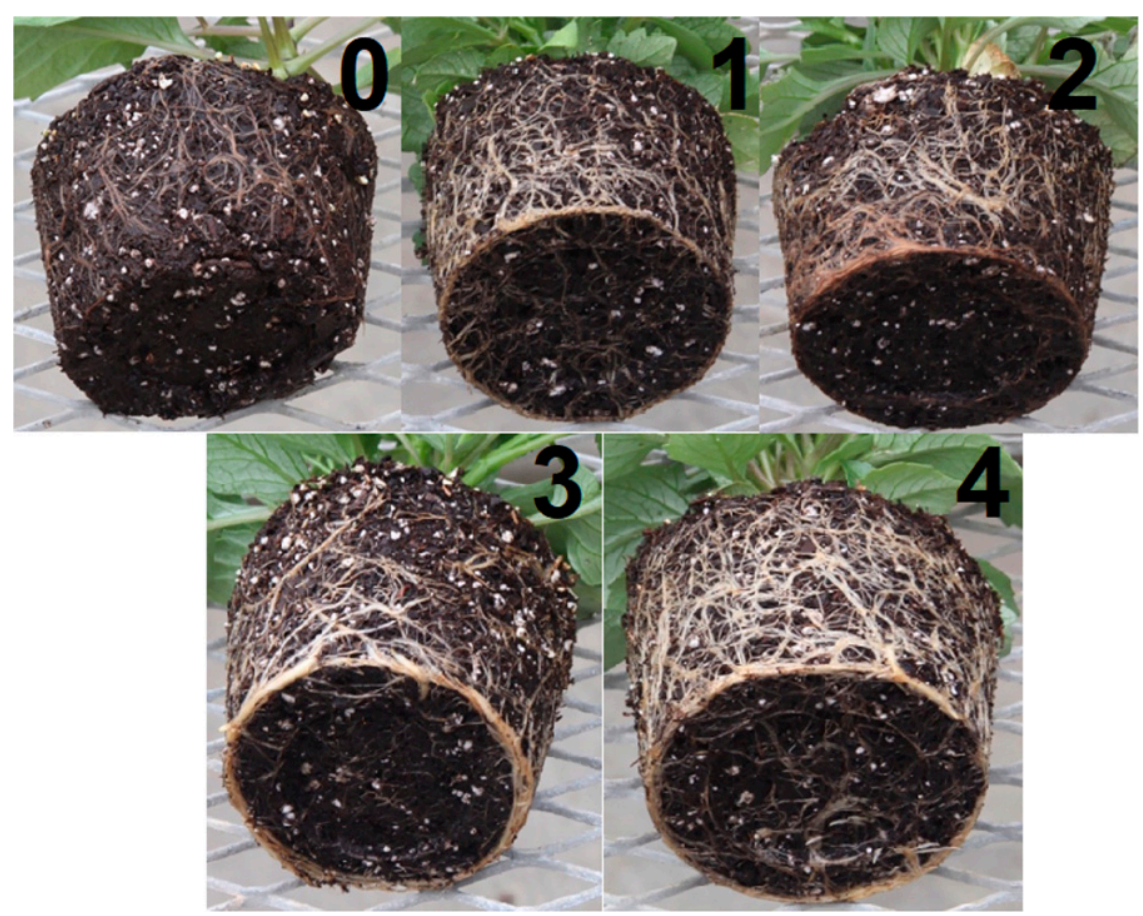

Fig. 1. Root rating scale (0-4), used to determine root quality in dahlia. Root ratings: $0=$ poor rooting (no roots; significant browning of roots, visibly in decline or absent), 1 = fair rooting (few roots; primarily white roots visible), $2=$ good rooting (roots wrapped around base of media, distributed throughout pot; no browning), 3 = very good rooting, (well-distributed roots, wrapped around pot, more thickly developed roots compared with thin), no browning, $4=$ excellent rooting (roots are highly developed, white, no signs of death). Research was conducted in glass houses at Manhattan, KS. 
for an individual experimental group, 25 uniform experimental plants from each cultivar were selected for the experiment. The Dalaya series was given an extra week in year 2 because it did not initially meet the threshold of sufficient rooting, which was determined to be when white, healthyappearing roots, were fully visible at the edge of the pot.

Using a water bath (Precision Model 186 and 260; Thermo Fisher Scientific, Waltham, MA), dahlias were exposed to supraoptimal RZT. There were five water bath temperature treatments: a control (room temperature $\approx 22^{\circ} \mathrm{C}$ ), and $35,40,45$, and $50^{\circ} \mathrm{C}$. These temperatures were selected based on a previous dahlia RZT study (P.A. Hammer, unpublished data).
Five plants or repetitions of each cultivar were assigned to each water bath temperature treatment. On treatment application day, plants were watered in the greenhouse and allowed to drain to pot capacity (no free-water in substrate) before water bath treatments. Plant growth parameter data (described later in this article) were recorded, and then dahlia pots were individually put into plastic bags. This was to prevent hot water from saturating the root zone. The bag tops were left open to aid in ventilation and allow heat and steam to escape. Plants were placed into water baths and heated until the root zone reached the desired treatment temperature. RZTs were measured using soil probes (5976 Soil Thermometer; Taylor, Rochester, NY) inserted into the root substrate midway between plant and edge of pot, roughly $l$ inch from the pot edge and 2 inches deep. After reaching the desired set-point temperature, plants were immediately removed from the water bath and plastic bag. Control plants were placed in plastic bags and in a room-temperature water bath for $2 \mathrm{~h}$, the average time it took to reach the desired supraoptimal temperatures of other treatments. After plants were treated, they were placed back into the greenhouse and maintained for 4 weeks posttreatment with root ratings and other plant observations taken once per week.

Data collection. Dahlia plants were carefully removed from their pots to assess root health based on an

Table 2. Significance levels of main effects and interactions for dahlia cultivar growth measurements observed in year 1 ( 6 Feb. to 25 May 2019) and year 2 (2 Feb. to 22 May 2020). Research was conducted in glass houses at Manhattan, KS.

\begin{tabular}{|c|c|c|c|c|c|c|c|}
\hline \multirow[b]{3}{*}{ Year 1} & \multicolumn{7}{|c|}{ Cultivar } \\
\hline & Red and White & Shiva & Yogi & Sunset & Tabasco & Veracruz & Sincerity \\
\hline & \multicolumn{7}{|c|}{ Significance $(P)$} \\
\hline \multicolumn{8}{|l|}{ Root rating } \\
\hline Temperature treatment & $<0.0001$ & $<0.0001$ & $<0.0001$ & $<0.0001$ & 0.0032 & $<0.0001$ & 0.0012 \\
\hline Observation week & $<0.0001$ & $<0.0001$ & 0.0008 & $<0.0001$ & $<0.0001$ & $<0.0001$ & $<0.0001$ \\
\hline Treatment $\times$ Week & 0.2401 & 0.0418 & 0.1693 & 0.3510 & 0.0005 & 0.0039 & 0.3154 \\
\hline \multicolumn{8}{|l|}{ Overall plant height } \\
\hline Temperature treatment & $<0.0001$ & 0.0014 & $<0.0001$ & 0.0269 & $<0.0001$ & 0.0036 & $<0.0001$ \\
\hline Observation week & $<0.0001$ & $<0.0001$ & $<0.0001$ & $<0.0001$ & $<0.0001$ & $<0.0001$ & $<0.0001$ \\
\hline Treatment $\times$ Week & 0.0009 & 0.0644 & 0.2875 & 0.0794 & 0.1562 & 0.7510 & 0.1873 \\
\hline \multicolumn{8}{|l|}{ Flower development rating } \\
\hline Temperature treatment & $<0.0001$ & 0.5847 & $<0.0001$ & 0.6840 & 0.1267 & $<0.0001$ & $-{ }^{\mathrm{z}}$ \\
\hline Observation week & $<0.0001$ & $<0.0001$ & $<0.0001$ & $<0.0001$ & $<0.0001$ & $<0.0001$ & - \\
\hline Treatment $\times$ Week & $<0.0001$ & 0.9992 & $<0.0001$ & 0.8984 & 0.1098 & 0.0882 & - \\
\hline \multicolumn{8}{|l|}{ Foliage rating } \\
\hline Temperature treatment & $<0.0001$ & $<0.0001$ & $<0.0001$ & 0.1003 & - & 0.2018 & 0.0007 \\
\hline Observation week & 0.0051 & 0.0923 & 0.0018 & 0.5603 & - & 0.1824 & 0.6658 \\
\hline Treatment $\times$ Week & 0.0019 & 0.3784 & 0.2622 & 0.7365 & - & 0.8433 & 0.9619 \\
\hline \multicolumn{8}{|l|}{ Year 2} \\
\hline \multicolumn{8}{|l|}{ Root rating } \\
\hline Temperature treatment & $<0.0001$ & $<0.0001$ & $<0.0001$ & $<0.0001$ & $<0.0001$ & $<0.0001$ & $<0.0001$ \\
\hline Observation week & $<0.0001$ & $<0.0001$ & 0.0079 & $<0.0001$ & $<0.0001$ & $<0.0001$ & $<0.0001$ \\
\hline Treatment $\times$ Week & 0.0091 & 0.0045 & 0.0009 & $<0.0001$ & 0.0147 & $<0.0001$ & 0.0686 \\
\hline \multicolumn{8}{|l|}{ Overall plant height } \\
\hline Temperature treatment & $<0.0001$ & $<0.0001$ & $<0.0001$ & $<0.0001$ & $<0.0001$ & $<0.0001$ & $<0.0001$ \\
\hline Observation week & $<0.0001$ & $<0.0001$ & $<0.0001$ & $<0.0001$ & $<0.0001$ & $<0.0001$ & $<0.0001$ \\
\hline Treatment $\times$ Week & 0.4741 & 0.0231 & 0.3789 & 0.0006 & 0.1964 & 0.2127 & 0.5381 \\
\hline \multicolumn{8}{|l|}{ Flower development rating } \\
\hline Temperature treatment & 0.0004 & $<0.0001$ & 0.2753 & $<0.0001$ & $<0.0001$ & $<0.0001$ & $<0.0001$ \\
\hline Observation week & $<0.0001$ & $<0.0001$ & $<0.0001$ & $<0.0001$ & $<0.0001$ & $<0.0001$ & $<0.0001$ \\
\hline Treatment $\times$ Week & 0.2283 & 0.0005 & 0.3216 & 0.0003 & 0.3546 & 0.0001 & $<0.0001$ \\
\hline \multicolumn{8}{|l|}{ Foliage rating } \\
\hline Temperature treatment & 0.0172 & $<0.0001$ & 0.0235 & $<0.0001$ & 0.0021 & $<0.0001$ & $<0.0001$ \\
\hline Observation week & 0.7459 & $<0.0001$ & 0.0235 & 0.0241 & 0.4868 & 0.4751 & 0.3643 \\
\hline Treatment $\times$ Week & 0.9891 & 0.0002 & 0.1410 & 0.0036 & 0.1740 & 0.5864 & 0.8156 \\
\hline
\end{tabular}

${ }^{\mathrm{z}}$ Dashes represent absence of probability value; statistical analysis was not performed due to zero variability. 
established root ratings scale (Fig. 1). Root ratings were defined as $0=$ poor rooting (no roots; significant browning of roots, visibly in decline or absent), 1 = fair rooting (few roots; primarily white roots visible), $2=$ good rooting (roots wrapped around base of media, distributed throughout pot; no browning), $3=$ very good rooting (well-distributed roots, wrapped around pot, more thickly developed roots compared with thin), no browning, and $4=$ excellent rooting (roots are highly developed, white, no signs of death). Overall plant height was recorded from bottom of the pot to top-most foliage growth. Flower development ratings were also collected and were based on a qualitative scale of 0 to $3: 0=$ no visible flower bud, $1=$ visible flower bud, 2 = flower bud with outer petals showing color, and $3=$ inflorescence open with ray florets expanded. Finally, plants were also evaluated for foliage quality. Foliage ratings were assigned using a scale of 0 to $4: 0=$ dead/completely brown leaves, $1=$ more than $60 \%$ brown with green still on leaves, $2=$ drooping/ flagging leaves with a less than $50 \%$ brown or graying, $3=$ drooping/flagging leaves, but otherwise green leaves, and $4=$ turgid, healthy, bright green leaves.

EXPERIMENTAL DESIGN AND STATISTICAL analysis. The experimental design was a completely randomized design. Statistical analyses were performed using JMP Pro (version 14; SAS Institute, Cary, NC) statistical software. An analysis of variance using the general linear model was used and mean separations were determined using Tukey's honestly significant difference test at a significance level of $\alpha=0.05$. In our statistical analysis, we found both year and cultivar to be significant for root rating. To better understand individual cultivar reactions to the treatments, we analyzed each year and cultivar separately.

\section{Results}

Root ratings. The main effects of temperature treatment and observation week were significant in all cultivars for both years (Table 2). In both years, as temperature treatments increased, root ratings decreased, except for 'Melody Sincerity' (Table 3). Root ratings generally decreased for cultivars in both years 1 week after water bath treatments, although not all were significant (Table 3). By the end of the experiment, at 4 weeks posttreatment, XXL cultivars had increased root ratings both years and Melody Sincerity in year 2 (Table 3). The interaction of main effects was variable. In year 1 , they were significant for 'Dalaya Shiva', 'XXL Tabasco', and 'XXL Veracruz' and in year 2 for all cultivars except for Melody Sincerity (Table 2). Root rating decreases, although not significant, were

Table 3. Effects of water bath temperature treatments and observation weeks on root ratings for dahlia cultivars in year 1 (6 Feb. to 25 May 2019). Research was conducted in glass houses at Manhattan, KS.

\begin{tabular}{|c|c|c|c|c|c|c|c|}
\hline \multirow[b]{2}{*}{ Year 1} & \multicolumn{7}{|c|}{ Cultivar } \\
\hline & \multicolumn{7}{|c|}{ Root rating $(0-4 \text { scale })^{\mathrm{z}}$} \\
\hline \multicolumn{8}{|c|}{ Temp treatment $\left({ }^{\circ} \mathrm{C}\right)^{\mathrm{y}}$} \\
\hline Control (22) & $0.60 \mathrm{a}^{\mathrm{x}}$ & $0.65 \mathrm{a}$ & $1.16 \mathrm{a}$ & $1.89 \mathrm{a}$ & $1.31 \mathrm{a}$ & $2.98 \mathrm{a}$ & $0.98 \mathrm{ab}$ \\
\hline 35 & $0.45 \mathrm{a}$ & $0.76 \mathrm{a}$ & $1.20 \mathrm{a}$ & $2.09 \mathrm{a}$ & $0.96 \mathrm{ab}$ & $2.83 \mathrm{ab}$ & $1.29 \mathrm{a}$ \\
\hline 50 & $0.20 \mathrm{~b}$ & $0.31 \mathrm{c}$ & $0.63 \mathrm{bc}$ & $0.90 \mathrm{~b}$ & $0.78 \mathrm{~b}$ & $1.65 \mathrm{c}$ & $0.83 \mathrm{~b}$ \\
\hline \multicolumn{8}{|c|}{ Observation week } \\
\hline Pretreatment & $0.64 \mathrm{a}$ & $0.62 \mathrm{ab}$ & $0.79 \mathrm{ab}$ & $1.34 \mathrm{bc}$ & $0.60 \mathrm{c}$ & $2.26 \mathrm{~b}$ & $0.86 \mathrm{bc}$ \\
\hline 1 & $0.28 \mathrm{c}$ & $0.30 \mathrm{c}$ & $0.62 \mathrm{~b}$ & $0.89 \mathrm{c}$ & $0.61 \mathrm{c}$ & $1.28 \mathrm{c}$ & $0.59 \mathrm{c}$ \\
\hline 2 & $0.37 \mathrm{bc}$ & $0.48 \mathrm{bc}$ & $0.97 \mathrm{a}$ & $1.57 \mathrm{~b}$ & $0.96 \mathrm{bc}$ & $2.10 \mathrm{~b}$ & $1.00 \mathrm{~b}$ \\
\hline 3 & $0.39 \mathrm{bc}$ & $0.56 \mathrm{ab}$ & $0.93 \mathrm{ab}$ & $1.91 \mathrm{ab}$ & $1.16 \mathrm{~b}$ & $2.99 \mathrm{a}$ & $1.41 \mathrm{a}$ \\
\hline \multicolumn{8}{|l|}{ Year 2} \\
\hline \multicolumn{8}{|c|}{ Temp treatment $\left({ }^{\circ} \mathrm{C}\right)$} \\
\hline Control (22) & $0.80 \mathrm{a}$ & $0.82 \mathrm{ab}$ & $1.48 \mathrm{a}$ & $3.14 \mathrm{a}$ & $1.40 \mathrm{a}$ & $3.68 \mathrm{a}$ & $1.36 \mathrm{ab}$ \\
\hline 35 & $0.52 \mathrm{~b}$ & $0.98 \mathrm{a}$ & $1.66 \mathrm{a}$ & $3.34 \mathrm{a}$ & $1.06 \mathrm{ab}$ & $3.32 \mathrm{ab}$ & $1.74 \mathrm{a}$ \\
\hline 40 & $0.46 \mathrm{~b}$ & $0.72 \mathrm{ab}$ & $1.12 \mathrm{~b}$ & $2.36 \mathrm{~b}$ & $1.40 \mathrm{a}$ & $2.78 \mathrm{~b}$ & $1.72 \mathrm{a}$ \\
\hline 45 & $0.56 \mathrm{ab}$ & $0.54 \mathrm{bc}$ & $0.66 \mathrm{c}$ & $2.60 \mathrm{~b}$ & $1.20 \mathrm{a}$ & $3.02 \mathrm{~b}$ & $1.38 \mathrm{ab}$ \\
\hline 50 & $0.30 \mathrm{~b}$ & $0.36 \mathrm{c}$ & $1.02 \mathrm{~b}$ & $1.06 \mathrm{c}$ & $0.62 \mathrm{~b}$ & $1.82 \mathrm{c}$ & $0.98 \mathrm{a}$ \\
\hline \multicolumn{8}{|c|}{ Observation week } \\
\hline Pretreatment & $1.10 \mathrm{a}$ & $0.90 \mathrm{a}$ & $1.18 \mathrm{ab}$ & $2.34 \mathrm{c}$ & $0.74 \mathrm{~b}$ & $3.20 \mathrm{a}$ & $1.08 \mathrm{bc}$ \\
\hline 1 & $0.46 \mathrm{~b}$ & $0.48 \mathrm{~b}$ & $0.98 \mathrm{~b}$ & $1.30 \mathrm{~d}$ & $0.60 \mathrm{~b}$ & $1.70 \mathrm{~b}$ & $0.74 \mathrm{c}$ \\
\hline 2 & $0.36 \mathrm{~b}$ & $0.52 \mathrm{~b}$ & $1.28 \mathrm{ab}$ & $2.56 \mathrm{bc}$ & $1.26 \mathrm{a}$ & $3.04 \mathrm{a}$ & $1.26 \mathrm{~b}$ \\
\hline 3 & $0.26 \mathrm{~b}$ & $0.62 \mathrm{ab}$ & $1.12 \mathrm{ab}$ & $2.96 \mathrm{ab}$ & $1.42 \mathrm{a}$ & $3.28 \mathrm{a}$ & $1.96 \mathrm{a}$ \\
\hline 4 & $0.45 \mathrm{~b}$ & $0.90 \mathrm{a}$ & $1.38 \mathrm{a}$ & $3.34 \mathrm{a}$ & $1.66 \mathrm{a}$ & $3.40 \mathrm{a}$ & $2.14 \mathrm{a}$ \\
\hline
\end{tabular}

${ }^{\mathrm{z}}$ Rating is an average of 25 repetitions: $0=$ poor rooting (no roots; significant browning of roots, visibly in decline or absent), $\mathrm{l}=$ fair rooting (few roots; primarily white roots visible), 2 = good rooting (roots wrapped around base of media, distributed throughout pot; no browning), $3=$ very good rooting, (well-distributed roots, wrapped around pot, more thickly developed roots compared with thin), no browning, $4=$ excellent rooting (roots are highly developed, white, no signs of death). ${ }^{\mathrm{y}}\left(1.8 \times{ }^{\circ} \mathrm{C}\right)+32={ }^{\circ} \mathrm{F}$.

${ }^{\mathrm{x}}$ Values followed by different letters are significantly different within a cultivar, across treatment or week using Tukey's mean separation test at $P \leq 0.05$. 
observed in nearly all cultivars, primarily in the higher temperature treatments $\left(40,45\right.$, and $\left.50^{\circ} \mathrm{C}\right)$ from pretreatment observations to 1 week posttreatment both years (Tables 4 and 5 ). Root ratings decreased significantly $\mathrm{l}$ week after pretreatment observations at 45 and $50^{\circ} \mathrm{C}$ for 'Dalaya Red and White' in year 2 (Table 4 ), and for 'XXL Sunset' and 'XXL Veracruz' (Table 5, Fig. 2). In some cultivars, root rating average remained unchanged or increased, although not significantly for control, and $35^{\circ} \mathrm{C}$ l week after treatment. XXL series cultivars showed an increase in root ratings from 1 week posttreatment to 4 weeks posttreatment; XXL
Sunset $\left(45\right.$ and $\left.50^{\circ} \mathrm{C}\right)$, XXL Tabasco (control, $50^{\circ} \mathrm{C}$ ), and XXL Veracruz (all treatments) (Table 5), in year 1 . Similarly, in year 2, root ratings increased significantly from 1 week posttreatment to 4 weeks posttreatment at $50^{\circ} \mathrm{C}$ in 'Dalaya Yogi' (Table $4)$; at 40,45 , and $50^{\circ} \mathrm{C}$ for 'XXL Sunset' and 'XXL Veracruz' and $45^{\circ} \mathrm{C}$ for 'XXL Tabasco' (Table 5); and at 40, 45 , and $50^{\circ} \mathrm{C}$ for 'Melody Sincerity' (Table 5).

Overall Plant Height. Overall plant heights were significantly different for the main effects of temperature treatment and observation week for all cultivars in both year 1 and year 2 (Table 2). The only significant interaction in year 1 was observed in 'Dalaya Red and White' (Table 2). A significant interaction between temperature treatment and observation week was observed in year 2 in 'Dalaya Shiva' and 'XXL Sunset' (Table 2). Cultivars Dalaya Yogi and XXL Tabasco that were exposed to $45^{\circ} \mathrm{C}$ resulted in shorter plants compared with the control in both years, and Melody Sincerity in year 1 (Table 6). Similarly, plants treated at $50^{\circ} \mathrm{C}$ resulted in significantly shorter plants compared with the control for all cultivars except for XXL Veracruz in year 1 and for all cultivars in year 2 (Table 6). Overall plant

Table 4. The effect of temperature treatments on root rating, posttreatment, over a period of 4 observation weeks on dahlia Dalaya series for year 1 (6 Feb. to 25 May 2019) and year 2 (2 Feb. to 9 Apr. 2020). Research was conducted in glass houses at Manhattan, KS.

\begin{tabular}{|c|c|c|c|c|c|c|}
\hline \multirow[b]{2}{*}{ Cultivar } & \multicolumn{6}{|c|}{ Observation week } \\
\hline & Temp treatment $\left({ }^{\circ} \mathbf{C}\right)^{\mathbf{z}}$ & Pretreatment & 1 & 2 & 3 & 4 \\
\hline \multirow{4}{*}{$\frac{\text { Year } 1}{\text { Red and White }}$} & \multicolumn{6}{|c|}{ Root rating $(0-4 \text { scale })^{\mathrm{y}}$} \\
\hline & 35 & $0.10 \mathrm{bc}$ & $0.20 \mathrm{bc}$ & $0.50 \mathrm{abc}$ & $0.50 \mathrm{abc}$ & $0.60 \mathrm{ab}$ \\
\hline & 40 & $0.20 \mathrm{bc}$ & $0.10 \mathrm{bc}$ & $0.40 \mathrm{abc}$ & $0.50 \mathrm{abc}$ & $0.50 \mathrm{abc}$ \\
\hline & 45 & $0.20 \mathrm{bc}$ & $0.10 \mathrm{bc}$ & $0.60 \mathrm{ab}$ & $0.80 \mathrm{a}$ & $0.80 \mathrm{a}$ \\
\hline \multirow[t]{5}{*}{ Shiva } & Control (22) & $0.60 \mathrm{abc}$ & $0.20 \mathrm{abc}$ & $0.50 \mathrm{abc}$ & $0.50 \mathrm{abc}$ & $0.60 \mathrm{abc}$ \\
\hline & 35 & $0.30 \mathrm{abc}$ & $0.20 \mathrm{abc}$ & $0.70 \mathrm{ab}$ & $0.70 \mathrm{ab}$ & $0.80 \mathrm{a}$ \\
\hline & 40 & $0.20 \mathrm{abc}$ & $0.10 \mathrm{bc}$ & $0.60 \mathrm{abc}$ & $0.60 \mathrm{abc}$ & $0.70 \mathrm{ab}$ \\
\hline & 45 & $0.30 \mathrm{abc}$ & $0.10 \mathrm{bc}$ & $0.30 \mathrm{abc}$ & $0.40 \mathrm{abc}$ & $0.40 \mathrm{abc}$ \\
\hline & 50 & $0.30 \mathrm{abc}$ & $0.00 \mathrm{c}$ & $0.10 \mathrm{bc}$ & $0.30 \mathrm{abc}$ & $0.60 \mathrm{abc}$ \\
\hline Yogi & Control (22) & $0.40 \mathrm{abc}$ & $0.40 \mathrm{abc}$ & $1.00 \mathrm{abc}$ & $1.20 \mathrm{ab}$ & $1.20 \mathrm{ab}$ \\
\hline \multicolumn{7}{|l|}{ Year 2} \\
\hline \multirow[t]{5}{*}{ Red and White } & Control (22) & $1.10 \mathrm{ab}$ & $1.00 \mathrm{abc}$ & $0.90 \mathrm{abcd}$ & $0.40 \mathrm{bcde}$ & 0.60 abcde \\
\hline & 35 & $1.00 \mathrm{abc}$ & 0.60 abcde & 0.40 bcde & 0.20 cde & 0.40 bcde \\
\hline & 40 & $1.10 \mathrm{ab}$ & 0.60 abcde & 0.20 cde & 0.20 cde & 0.20 cde \\
\hline & 45 & $1.40 \mathrm{a}$ & $0.10 \mathrm{de}$ & $0.30 \mathrm{bcde}$ & 0.50 bcde & 0.50 bcde \\
\hline & 50 & $0.90 \mathrm{abcd}$ & $0.00 \mathrm{e}$ & $0.00 \mathrm{e}$ & $0.10 \mathrm{de}$ & 0.50 bcde \\
\hline \multirow[t]{4}{*}{ Shiva } & Control (22) & $0.90 \mathrm{abcd}$ & $0.80 \mathrm{abcd}$ & $0.80 \mathrm{abcd}$ & $0.70 \mathrm{abcd}$ & $0.90 \mathrm{abcd}$ \\
\hline & 35 & $1.20 \mathrm{a}$ & $1.20 \mathrm{a}$ & $1.00 \mathrm{abc}$ & $0.70 \mathrm{abcd}$ & $0.80 \mathrm{abcd}$ \\
\hline & 40 & $0.80 \mathrm{abcd}$ & $0.20 \mathrm{bcd}$ & $0.50 \mathrm{abcd}$ & $1.10 \mathrm{ab}$ & $1.00 \mathrm{abc}$ \\
\hline & 45 & $0.70 \mathrm{abcd}$ & $0.20 \mathrm{bcd}$ & $0.30 \mathrm{abcd}$ & $0.50 \mathrm{abcd}$ & $1.00 \mathrm{abc}$ \\
\hline
\end{tabular}

\footnotetext{
${ }^{\mathrm{z}}\left(1.8 \times{ }^{\circ} \mathrm{C}\right)+32={ }^{\circ} \mathrm{F}$.

${ }^{\mathrm{y}}$ Rating is an average of five repetitions: $0=$ poor rooting (no roots; significant browning of roots, visibly in decline or absent), $1=$ fair rooting (few roots; primarily white roots visible), 2 good rooting (roots wrapped around base of media, distributed throughout pot; no browning), $3=$ very good rooting, (well-distributed roots, wrapped around pot, more thickly developed roots compared with thin), no browning, $4=$ excellent rooting (roots are highly developed, white, no signs of death).

${ }^{\mathrm{x}}$ Values followed by different letters are significantly different within a cultivar, across treatment or week using Tukey's mean separation test at $P \leq 0.05$.
} 
height was significantly taller from the pretreatment observations (week 0 ) compared with 4 weeks posttreatment for all cultivars for both years (Table 6).

Flower Development. Temperature treatment significantly affected flower development ratings for cultivars Dalaya Red and White, Dalaya Yogi, and XXL Veracruz in year 1 and all cultivars in year 2 (Table 2, Fig. 3). Observation week had a significant effect for all cultivars both years (Table 2).
Significant interactions were observed, with the exceptions of, 'Dalaya Shiva' and all XXL cultivars in year 1 and for Dalaya Red and White, Dalaya Yogi, and XXL Tabasco, in year 2 (Table 2). Select cultivars showed flower

Table 5. The effect of temperature treatments on root rating, posttreatment, over a period of 4 observation weeks on dahlia XXL and Melody series for year 1 (22 Feb. to 25 May 2019) and year 2 (20 Feb. to 24 May 2020). Research was conducted in glass houses at Manhattan, KS.

\begin{tabular}{|c|c|c|c|c|c|c|}
\hline \multirow[b]{2}{*}{ Cultivar } & \multicolumn{6}{|c|}{ Observation week } \\
\hline & \multirow[t]{2}{*}{ Temp treatment $\left({ }^{\circ} \mathrm{C}\right)^{\mathrm{z}}$} & Pretreatment & 1 & 2 & 3 & 4 \\
\hline Year 1 & & \multicolumn{5}{|c|}{ Root rating $(0-4 \text { scale })^{\mathrm{y}}$} \\
\hline \multirow{3}{*}{ Sunset } & 35 & $0.40 \mathrm{ef}$ & 0.90 abcdef & 0.80 bcdef & $0.60 \mathrm{cdef}$ & $1.50 \mathrm{abcd}$ \\
\hline & 40 & $0.20 \mathrm{f}$ & $0.50 \mathrm{def}$ & $0.60 \mathrm{cdef}$ & 0.60 cdef & 1.40 abcde \\
\hline & 45 & 0.40 ef & $0.30 \mathrm{f}$ & $0.60 \mathrm{cdef}$ & $1.60 \mathrm{abc}$ & $1.70 \mathrm{ab}$ \\
\hline \multirow[t]{5}{*}{ Tabasco } & Control (22) & $0.50 \mathrm{c}$ & $0.90 \mathrm{c}$ & $1.00 \mathrm{c}$ & $1.10 \mathrm{bc}$ & $2.60 \mathrm{a}$ \\
\hline & 35 & $0.60 \mathrm{c}$ & $1.20 \mathrm{abc}$ & $0.60 \mathrm{c}$ & $0.70 \mathrm{c}$ & $1.20 \mathrm{abc}$ \\
\hline & 40 & $0.40 \mathrm{c}$ & $0.50 \mathrm{c}$ & $0.50 \mathrm{c}$ & $0.60 \mathrm{c}$ & $0.80 \mathrm{c}$ \\
\hline & 45 & $0.50 \mathrm{c}$ & $0.40 \mathrm{c}$ & $0.50 \mathrm{c}$ & $1.30 \mathrm{abc}$ & $1.50 \mathrm{abc}$ \\
\hline & 50 & $0.60 \mathrm{c}$ & $0.10 \mathrm{c}$ & $0.70 \mathrm{c}$ & $0.80 \mathrm{c}$ & $2.50 \mathrm{ab}$ \\
\hline Veracruz & Control (22) & 1.70 bcdef & $1.60 \mathrm{bcdef}$ & 1.70 bcdef & $2.40 \mathrm{abcd}$ & $4.00 \mathrm{a}$ \\
\hline \multicolumn{7}{|l|}{ Melody } \\
\hline \multirow[t]{5}{*}{ Sincerity } & Control (22) & $0.50 \mathrm{abc}$ & $0.50 \mathrm{abc}$ & $0.60 \mathrm{abc}$ & $0.70 \mathrm{abc}$ & $0.70 \mathrm{abc}$ \\
\hline & 35 & $0.60 \mathrm{abc}$ & $0.80 \mathrm{abc}$ & $0.80 \mathrm{abc}$ & $1.00 \mathrm{ab}$ & $1.00 \mathrm{ab}$ \\
\hline & 40 & $0.70 \mathrm{abc}$ & $0.80 \mathrm{abc}$ & $0.60 \mathrm{abc}$ & $1.00 \mathrm{ab}$ & $1.20 \mathrm{a}$ \\
\hline & 45 & $0.70 \mathrm{abc}$ & $0.00 \mathrm{c}$ & $0.70 \mathrm{abc}$ & $0.80 \mathrm{abc}$ & $0.60 \mathrm{abc}$ \\
\hline & 50 & $0.70 \mathrm{abc}$ & $0.10 \mathrm{bc}$ & $1.00 \mathrm{ab}$ & $0.80 \mathrm{abc}$ & $0.80 \mathrm{abc}$ \\
\hline \multicolumn{7}{|l|}{ Year 2} \\
\hline \multirow[t]{3}{*}{ Sunset } & Control (22) & $2.60 \mathrm{abcd}$ & $2.50 \mathrm{abcd}$ & $3.30 \mathrm{abc}$ & $3.50 \mathrm{a}$ & $3.80 \mathrm{a}$ \\
\hline & 35 & $2.80 \mathrm{abcd}$ & $3.10 \mathrm{abcd}$ & $3.40 \mathrm{ab}$ & $3.50 \mathrm{a}$ & $3.80 \mathrm{a}$ \\
\hline & 40 & $1.80 \mathrm{defg}$ & $0.50 \mathrm{fgh}$ & $2.90 \mathrm{abcd}$ & $3.30 \mathrm{abc}$ & $3.30 \mathrm{abc}$ \\
\hline \multirow{4}{*}{ Veracruz } & 35 & $3.10 \mathrm{abc}$ & $3.20 \mathrm{ab}$ & $3.50 \mathrm{ab}$ & $3.40 \mathrm{ab}$ & $3.40 \mathrm{ab}$ \\
\hline & 40 & $2.50 \mathrm{abcd}$ & $0.90 \mathrm{de}$ & $3.30 \mathrm{ab}$ & $3.60 \mathrm{ab}$ & $3.60 \mathrm{ab}$ \\
\hline & 45 & $3.70 \mathrm{ab}$ & $0.40 \mathrm{e}$ & $3.40 \mathrm{ab}$ & $3.70 \mathrm{ab}$ & $3.90 \mathrm{a}$ \\
\hline & 50 & $3.20 \mathrm{ab}$ & $0.30 \mathrm{e}$ & $1.20 \mathrm{cde}$ & 1.90 bcde & $2.50 \mathrm{abcd}$ \\
\hline \multicolumn{7}{|l|}{ Melody } \\
\hline \multirow[t]{5}{*}{ Sincerity } & Control (22) & $0.80 \mathrm{cde}$ & 1.30 abcde & 1.30 abcde & $1.70 \mathrm{abc}$ & $1.70 \mathrm{abc}$ \\
\hline & 35 & 1.20 abcde & 1.20 abcde & 1.40 abcde & $2.30 \mathrm{a}$ & $2.60 \mathrm{ab}$ \\
\hline & 40 & 1.20 abcde & 0.90 bcde & $1.60 \mathrm{abcd}$ & $2.40 \mathrm{a}$ & $2.50 \mathrm{a}$ \\
\hline & 45 & 0.90 bcde & $0.20 \mathrm{de}$ & 1.40 abcde & $1.90 \mathrm{abc}$ & $2.50 \mathrm{a}$ \\
\hline & 50 & 1.30 abcde & $0.10 \mathrm{e}$ & $0.60 \mathrm{cde}$ & 1.20 abcde & $1.70 \mathrm{abc}$ \\
\hline
\end{tabular}

\footnotetext{
${ }^{\mathrm{z}}\left(1.8 \times{ }^{\circ} \mathrm{C}\right)+32={ }^{\circ} \mathrm{F}$.

${ }^{y}$ Rating is an average of five repetitions: $0=$ poor rooting (no roots; significant browning of roots, visibly in decline or absent), $1=$ fair rooting (few roots; primarily white roots visible), 2 = good rooting (roots wrapped around base of media, distributed throughout pot; no browning), $3=$ very good rooting, (well-distributed roots, wrapped around pot, more thickly developed roots compared with thin), no browning, $4=$ excellent rooting (roots are highly developed, white, no signs of death).

${ }^{\mathrm{x}}$ Values followed by different letters are significantly different within a cultivar, across treatment or week using Tukey's mean separation test at $P \leq 0.05$.
} 
development was significantly lower at $50{ }^{\circ} \mathrm{C}$ compared with control in both years. In year 1, only 'Dalaya Red and White' and 'Dalaya Yogi', had significantly lower flower development, and in year 2, all cultivars except for Dalaya Yogi, flower development was significantly reduced (Table 7). Flower development increased over time in plants at pretreatment observations compared with 4 weeks posttreatment in both years, except for 'Melody Sincerity' in year 1 (Table 7).

Foliage RATING. Foliage rating observations indicated significant differences in temperature treatment for all cultivars in both years, except, XXL Sunset and XXL Veracruz in year 1 (Table 2). In year 1, 'XXL Tabasco' did not decrease in foliage ratings after any treatment; therefore, no statistical data are presented (Table 2). Observation week only affected 'Dalaya Red and White', and 'Dalaya Yogi' in year 1 and 'Dalaya Shiva', 'Dalaya Yogi' and 'XXL Sunset' in year 2 (Table 2). The interaction of temperature treatment by observation week was significant for 'Dalaya Red and White' in year 1 and 'Dalaya Shiva' and 'XXL Sunset' in year 2 (Table 2). Generally, foliage ratings only differed from untreated plants for the plants treated at $50{ }^{\circ} \mathrm{C}$ (Table 8 ). Foliage rating decreased in all cultivars at $50{ }^{\circ} \mathrm{C}$ compared with control, but only Dalaya Red and White, Dalaya Shiva, Dalaya Yogi, and Melody Sincerity were significant (Table 8). Foliage quality decreased significantly from pretreatment observations to 1 week posttreatment only for 'Dalaya Shiva' and 'Dalaya Yogi' in year 1. 'Dalaya Shiva', 'Dalaya Yogi', and 'XXL Sunset' exhibited significant foliage rating decreases 1 week after treatment in year 2 (Table 8 ).

\section{Discussion}

In our experiments, we observed that supraoptimal RZT for $2 \mathrm{~h}$ can impact root zones and plant growth. Dahlia root injury, expressed as decreases in dahlia root rating, suggests that plant roots were damaged, but the absence of total plant death and subsequent root rating increases even with plants subjected to $50{ }^{\circ} \mathrm{C}$ RZTs indicates that plants can recover even when their root zones are injured. Observations of root rating decrease in the surveyed dahlia cultivars were not surprising; previous research showed that when root zones were exposed to temperatures $>40^{\circ} \mathrm{C}$, root quality was impacted, and death can occur (Ingram et al., 2015). We observed root rating decreases, especially above $40^{\circ} \mathrm{C}$, in our experiments, indicating a threshold for potential root damage, which was also reported by Ingram et al. (2015). In our potted dahlia study, our supraoptimal treatments $\left(40,45\right.$, and $\left.50^{\circ} \mathrm{C}\right)$ were sub-lethal. Observing an increase in root ratings over time indicates improved root quality as time passed, even after supraoptimal heat damage has occurred. These observations suggest that dahlias have some tolerance to high temperatures and can be resilient. Similar field observations of plant recovery were reported by Ingram et al. (2015) when looking at 'St. Mary' southern magnolia exposed to supraoptimal temperatures between 30 and $42^{\circ} \mathrm{C}$.

Inconsistent reaction to the supraoptimal RZT among different cultivars could be due to specific cultivar responsiveness to the temperature. Martin et al. (1991) similarly observed specific cultivar reaction differences in southern magnolia. For example, our research showed the Dalaya series, in general, developed poor, or low-vigor root growth in comparison with the XXL series (Tables 4 and 5, Fig. 4). Looking at the overall experiment, considering both years, the highest root rating average observed in the Dalaya series was in 'Dalaya Yogi' in year 2 , with a rating of $2.00,2$ weeks after plants were exposed to $35^{\circ} \mathrm{C}$ (Table 4, Fig. 5), whereas the highest root rating average observed in the XXL series was 4.0 in 'XXL Veracruz' at the control and $35^{\circ} \mathrm{C}$ treatments the last week of observation in year 1 (Table 5, Fig. 5). Cultivar differences made finding statistically significant decline in ratings difficult in the Dalaya series when pretreatment root ratings were low to begin the experiment and remained low, even in the control group 4 weeks posttreatment (Table 4 , Fig. 5). In early production in year 2, 'Dalaya Red and White' began to exhibit symptoms of virus (distorted growth, stunted foliage); however, we did not test for virus. In garden and exhibition dahlias, there has been increasing awareness of dahlia virus.

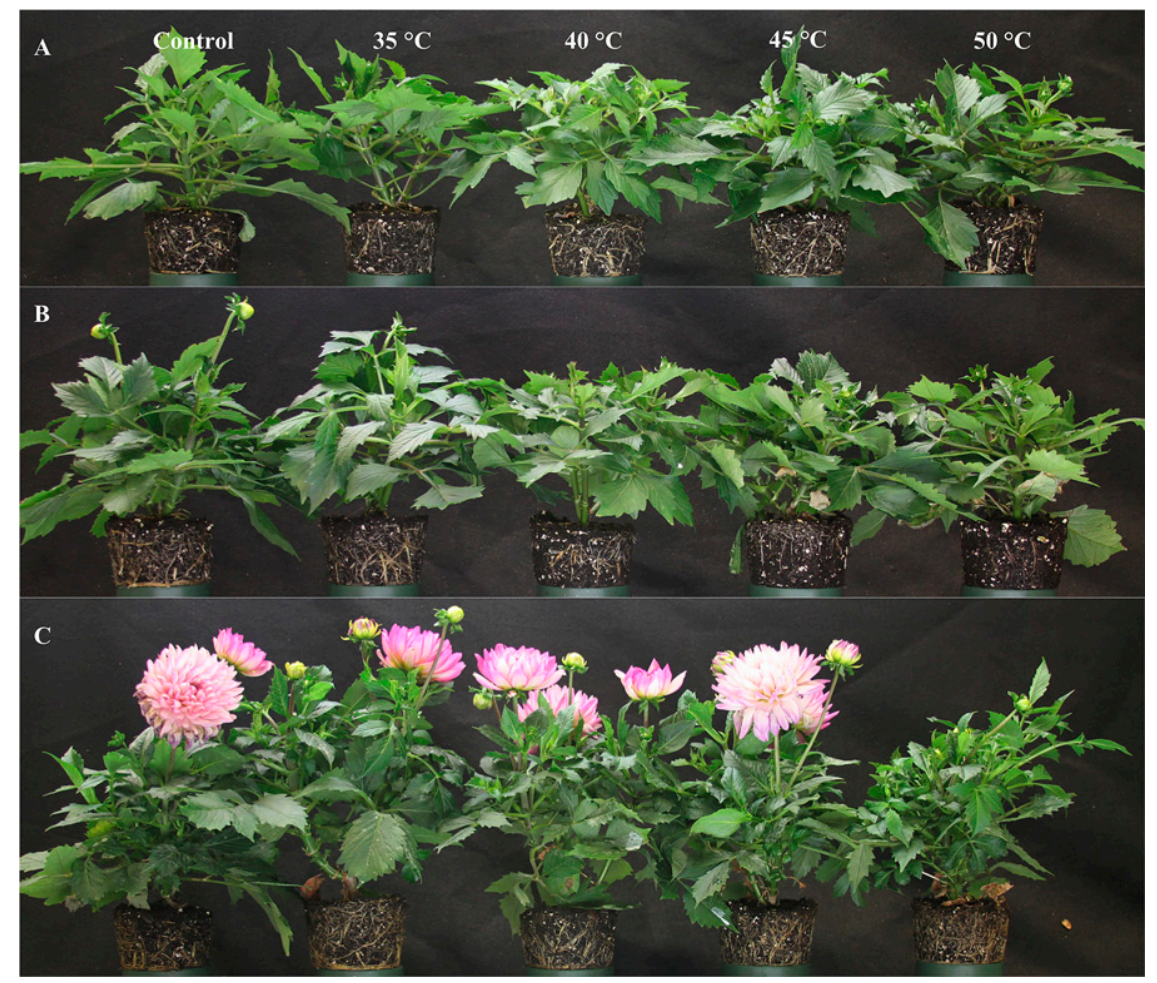

Fig. 2. Treatment comparisons for year 2 of 'XXL Veracruz' dahlia.

Representative plants from treatments are shown left to right in the photo; 22 (control), 35, 40, 45, 50 ${ }^{\circ} \mathrm{C}$ : (A) pretreatment observations, (B) 1 week posttreatment, and (C) 4 weeks posttreatment. Research was conducted in glass houses at Manhattan, KS, from $20 \mathrm{Feb}$. to 2 May 2020; $\left(1.8 \times{ }^{\circ} \mathrm{C}\right)+32={ }^{\circ} \mathrm{F}$. 
Table 6. Effects of temperature treatment and observation week on overall plant height for dahlia cultivars in year 1 (6 Feb. to 25 May 2019) and year 2 (2 Feb. to 22 May 2020). Research was conducted in glass houses at Manhattan, KS.

\begin{tabular}{|c|c|c|c|c|c|c|c|}
\hline \multirow[b]{3}{*}{ Year 1} & \multicolumn{7}{|c|}{ Cultivar } \\
\hline & Red and White & Shiva & Yogi & Sunset & Tabasco & Veracruz & Sincerity \\
\hline & \multicolumn{7}{|c|}{ Overall plant ht $(\mathrm{cm})^{\mathrm{z}}$} \\
\hline Control (22) & $17.26 \mathrm{ab}^{\mathrm{x}}$ & $16.04 \mathrm{a}$ & $16.56 \mathrm{a}$ & $17.34 \mathrm{a}$ & $18.08 \mathrm{a}$ & $19.78 \mathrm{ab}$ & $18.26 \mathrm{a}$ \\
\hline 35 & $17.04 \mathrm{ab}$ & $16.44 \mathrm{a}$ & $15.30 \mathrm{a}$ & $17.12 \mathrm{ab}$ & $17.18 \mathrm{ab}$ & $19.96 \mathrm{ab}$ & $18.72 \mathrm{a}$ \\
\hline 50 & $12.82 \mathrm{c}$ & $14.46 \mathrm{~b}$ & $12.21 \mathrm{~b}$ & $16.12 \mathrm{~b}$ & $16.12 \mathrm{c}$ & $19.46 \mathrm{ab}$ & $16.06 \mathrm{c}$ \\
\hline \multicolumn{8}{|l|}{ Week } \\
\hline Pretreatment & $13.90 \mathrm{c}$ & $13.40 \mathrm{c}$ & $12.44 \mathrm{c}$ & $13.52 \mathrm{e}$ & $13.82 \mathrm{e}$ & $18.10 \mathrm{c}$ & $16.08 \mathrm{c}$ \\
\hline 1 & $14.68 \mathrm{c}$ & $14.22 \mathrm{c}$ & $12.56 \mathrm{c}$ & $15.04 \mathrm{~d}$ & $15.14 \mathrm{~d}$ & $18.38 \mathrm{c}$ & $16.32 \mathrm{c}$ \\
\hline 2 & $15.02 \mathrm{c}$ & $14.70 \mathrm{c}$ & $12.99 \mathrm{c}$ & $16.86 \mathrm{c}$ & $16.76 \mathrm{c}$ & $19.36 \mathrm{bc}$ & $17.52 \mathrm{~b}$ \\
\hline Control (22) & $19.30 \mathrm{a}$ & $19.42 \mathrm{a}$ & $19.82 \mathrm{a}$ & $23.12 \mathrm{a}$ & $20.84 \mathrm{a}$ & $21.26 \mathrm{~b}$ & $21.18 \mathrm{a}$ \\
\hline 35 & $18.08 \mathrm{~b}$ & $20.41 \mathrm{a}$ & $20.42 \mathrm{a}$ & $22.41 \mathrm{ab}$ & $20.84 \mathrm{a}$ & $22.23 \mathrm{a}$ & $21.34 \mathrm{a}$ \\
\hline 40 & $18.50 \mathrm{ab}$ & $18.18 \mathrm{~b}$ & $18.18 \mathrm{~b}$ & $21.72 \mathrm{~b}$ & $20.18 \mathrm{a}$ & $20.78 \mathrm{~b}$ & $21.26 \mathrm{a}$ \\
\hline 45 & $18.80 \mathrm{ab}$ & $17.60 \mathrm{~b}$ & $17.60 \mathrm{~b}$ & $21.48 \mathrm{~b}$ & $18.76 \mathrm{~b}$ & $20.30 \mathrm{~b}$ & $19.66 \mathrm{a}$ \\
\hline 50 & $16.29 c$ & $16.30 \mathrm{c}$ & $16.30 \mathrm{c}$ & $18.98 \mathrm{c}$ & $17.80 \mathrm{~b}$ & $19.12 \mathrm{c}$ & $17.60 \mathrm{~b}$ \\
\hline \multicolumn{8}{|l|}{ Week } \\
\hline Pretreatment & $16.58 \mathrm{c}$ & $16.10 \mathrm{~d}$ & $16.58 \mathrm{e}$ & $18.96 \mathrm{~d}$ & $15.98 \mathrm{e}$ & $19.12 \mathrm{~d}$ & $18.28 \mathrm{~b}$ \\
\hline 1 & $17.72 \mathrm{bc}$ & $17.42 \mathrm{c}$ & $17.96 \mathrm{~d}$ & $20.36 c$ & $17.68 \mathrm{~d}$ & $19.96 \mathrm{~cd}$ & $18.46 \mathrm{~b}$ \\
\hline 2 & $18.44 \mathrm{ab}$ & $18.30 \mathrm{bc}$ & $19.16 \mathrm{c}$ & $21.34 \mathrm{c}$ & $19.46 \mathrm{c}$ & $20.66 \mathrm{bc}$ & $19.56 \mathrm{~b}$ \\
\hline 3 & $18.90 \mathrm{ab}$ & $19.38 \mathrm{ab}$ & $20.90 \mathrm{~b}$ & $22.78 \mathrm{~b}$ & $21.64 \mathrm{~b}$ & $21.66 \mathrm{ab}$ & $21.52 \mathrm{a}$ \\
\hline
\end{tabular}

${ }^{\mathrm{z}}$ Overall plant height measurement is average of 25 repetitions. Height was measured from bottom of pot to top of foliage canopy; $1 \mathrm{~cm}=0.3937 \mathrm{inch}$.

${ }^{\mathrm{y}}\left(1.8 \times{ }^{\circ} \mathrm{C}\right)+32={ }^{\circ} \mathrm{F}$.

${ }^{x}$ Values followed by different letters are significantly different within a cultivar, across treatment or week using Tukey's mean separation test at $P \leq 0.05$.

Increased research has been conducted to determine the extent of dahlia virus in the United States (Pappu et al., 2005). At least one commercial supplier indicated that their plant material is virus indexed for the vegetative cuttings sent to consumers (A. Carlson, personal communication). It is possible virus may have impacted overall plant development.

Significant decreases in root ratings that impact root health could also slow dahlia growth and flower development (Tables 6 and 7). For example, plant height was reduced significantly for all cultivars at $50^{\circ} \mathrm{C}$ and several cultivars at both 40 and $45^{\circ} \mathrm{C}$ in both years (Table 6). In 'XXL Veracruz', flowering was reduced at $50^{\circ} \mathrm{C}$ with only two replicates in bloom 4 weeks posttreatment, whereas the control had all five replicates in bloom (Table 7, Fig. 3). Most of the other cultivars also had lower flower development at $50^{\circ} \mathrm{C}$, except for Dalaya Yogi in year 2 (Table 7). Although roots may recover,

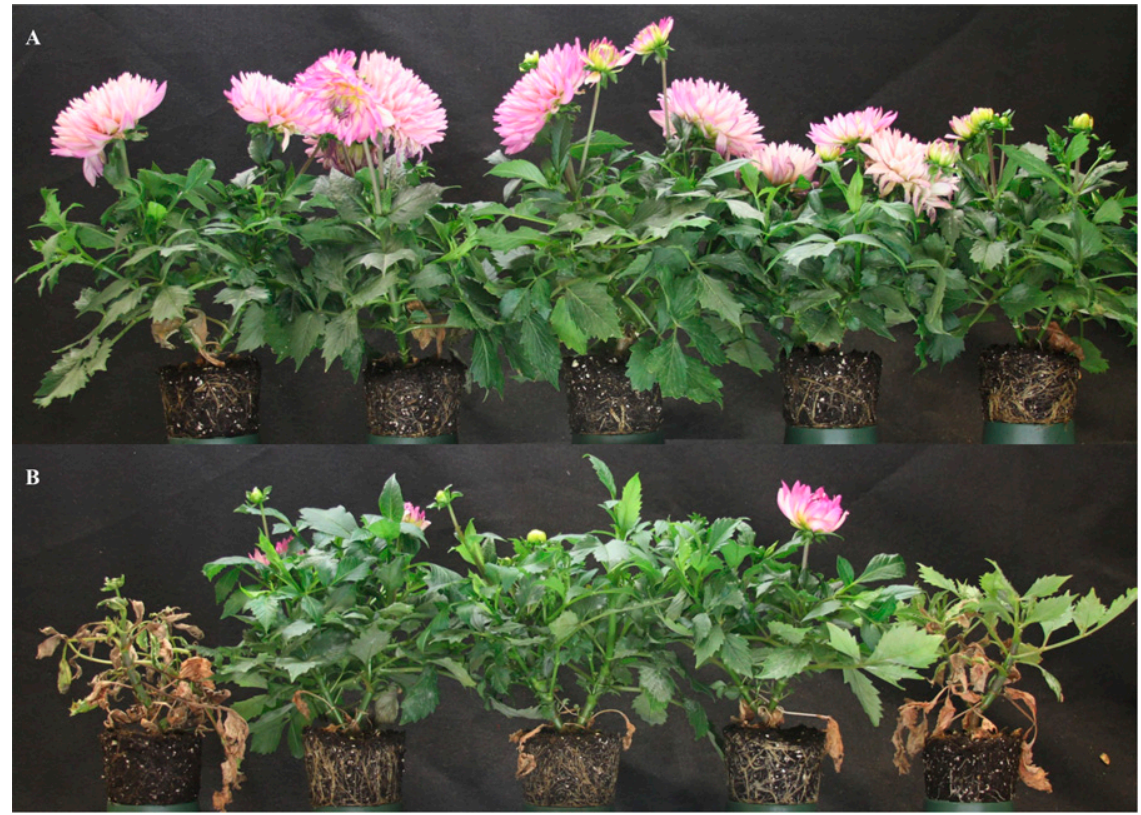

Fig. 3. Example of plant, root quality, and flowering response 4 weeks posttreatment in year 2 for 'XXL Veracruz' dahlia plants: (A) repetitions from control $\left[22{ }^{\circ} \mathrm{C}(71.6\right.$ $\left.{ }^{\circ} \mathrm{F}\right)$ ] treatment and $(\mathrm{B})$ repetitions from $50{ }^{\circ} \mathrm{C}\left(122.0^{\circ} \mathrm{F}\right)$ treatment. Research was conducted in glass houses from 20 Feb. to 2 May 2020 at Manhattan, KS. 
Table 7. Effects of temperature treatment and observation week on flower development ratings for dahlia cultivars in year 1 (6 Feb. to 25 May 2019) and year 2 (2 Feb. to 22 May 2020). Research was conducted in glass houses at Manhattan, KS.

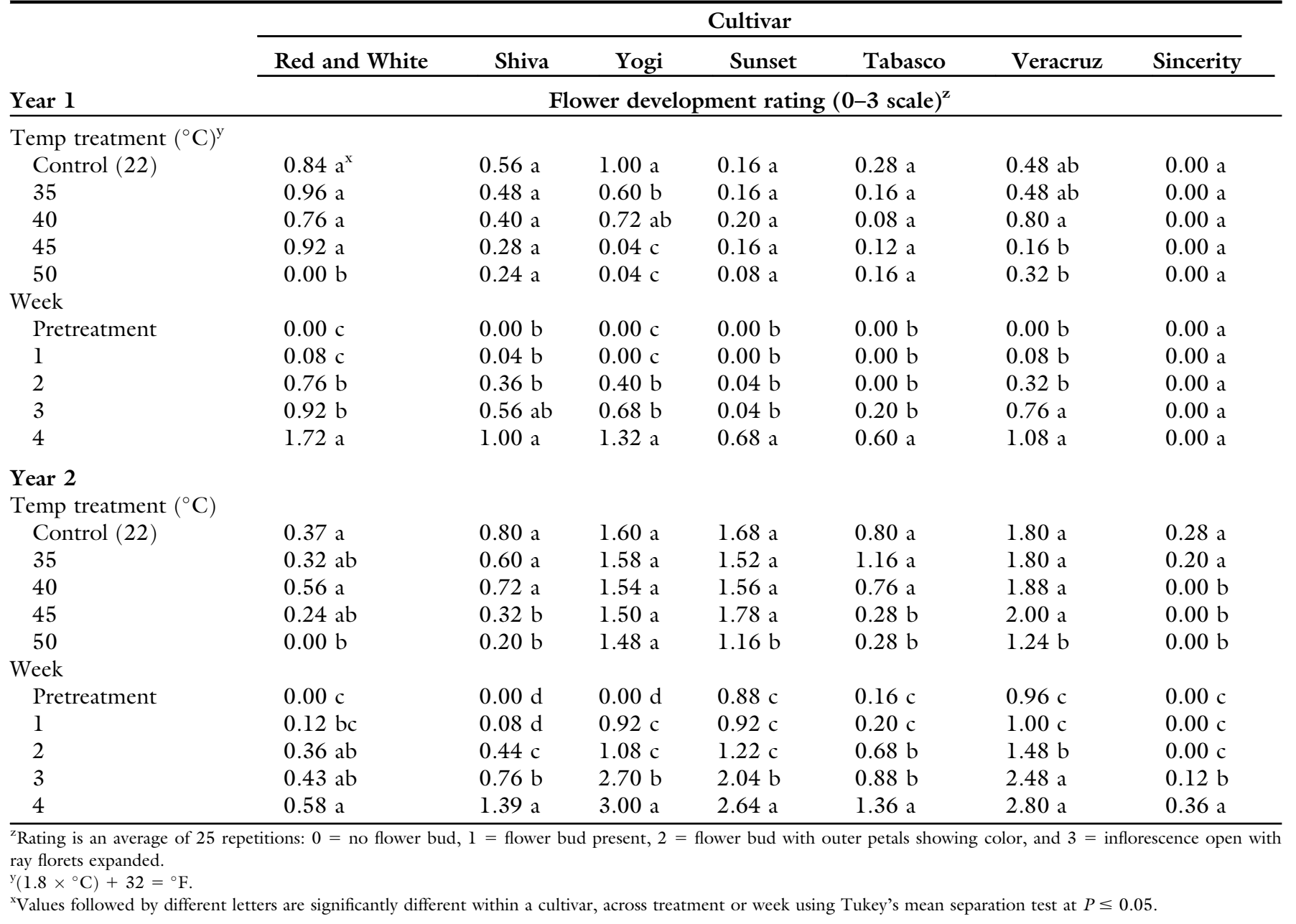

foliage damage or flower delay may prevent the plant from being ultimately marketable. In our experiments, we observed decreases in foliage ratings as the temperature treatments increased (Table 8). Even though these decreases were observed, we cannot say for certain that they were all caused by root injury. Some damage could be attributed to hot steam build up when treating the plants, even with ventilation provided.

Despite decreases in root ratings after exposure to supraoptimal RZTs (Tables 4 and 5 ), we were not successful in eliciting uniform symptoms of dahlia decline; however, there were limited repetitions that exhibited similar symptoms to dahlia decline and succumbed to death. The plant death observed was most likely due to individual cultivars having lower vigor roots or individual plants experiencing the supraoptimal temperature and simply not recovering like the others in the cultivar.
In addition to supraoptimal RZT as an explanation for dahlia decline, it is plausible that one may need to further consider root-zone pathogens. As previously described, dahlia decline symptoms include poor rooting or root decline, leaf-graying, and wilting foliage, then complete plant collapse (P.A. Hammer, personal communication). Diseases that would most likely fit these symptoms are root rots, especially pythium (Pythium). Pythium infection symptoms include stunted growth, dead root tips, sloughing of root cortex, and ultimately in severe cases, plant death (Beckerman, 2011). Considering that the most common greenhouse Pythium species (Pythium aphanidermatum, Pythium irregulare, and Pythium ultimum) are not able to survive at supraoptimal temperatures (i.e., $40^{\circ} \mathrm{C}$ and higher), it is plausible that dahlia decline observed at elevated RZT is not attributed to pythium due to the pathogen being killed off or at least greatly inhibited (Moorman and Daughtrey, 2002). If infection or reinfection were to occur after an elevated RZT situation, it might then be possible for pythium to infect and damage the root system. Although pythium could be a probable cause of dahlia decline, previous preliminary tests suggest that it is not a primary factor in dahlia decline (P.A. Hammer, personal communication). In our study, we did not find any evidence of pathogen interference in our treatments, including the limited number of plants that died. We can make this assertion because there was no consistent, widespread death in our supraoptimal treatments, and there was no death in the control treatment. If a pathogen like pythium had infected our initial rooted cuttings, we would have observed root decline in more than just our supraoptimal treatments; that is, our control plants would have also declined. Moreover, to help 
Table 8. Effects of temperature treatment and week on foliage rating for dahlia cultivars in year 1 (6 Feb. to $25 \mathrm{May}$ 2019) and year 2 (2 Feb. to 22 May 2020). Research was conducted in glass houses at Manhattan, KS.

\begin{tabular}{|c|c|c|c|c|c|c|c|}
\hline \multirow[b]{3}{*}{ Year 1} & \multicolumn{7}{|c|}{ Cultivar } \\
\hline & Red and White & Shiva & Yogi & Sunset & Tabasco & Veracruz & Sincerity \\
\hline & \multicolumn{7}{|c|}{ Foliage rating $(0-4 \text { scale })^{\mathrm{z}}$} \\
\hline \multicolumn{8}{|c|}{ Temp treatment $\left({ }^{\circ} \mathrm{C}\right)^{\mathrm{y}}$} \\
\hline Control (22) & $4.00 \mathrm{a}^{\mathrm{x}}$ & $4.00 \mathrm{a}$ & $4.00 \mathrm{a}$ & $4.00 \mathrm{a}$ & $4.00 \mathrm{a}$ & $4.00 \mathrm{a}$ & $4.00 \mathrm{a}$ \\
\hline 35 & $4.00 \mathrm{a}$ & $4.00 \mathrm{a}$ & $4.00 \mathrm{a}$ & $4.00 \mathrm{a}$ & $4.00 \mathrm{a}$ & $4.00 \mathrm{a}$ & $4.00 \mathrm{a}$ \\
\hline 50 & $1.92 \mathrm{~b}$ & $3.36 \mathrm{~b}$ & $2.08 \mathrm{~b}$ & $3.92 \mathrm{a}$ & $4.00 \mathrm{a}$ & $3.76 \mathrm{a}$ & $3.48 \mathrm{~b}$ \\
\hline \multicolumn{8}{|c|}{ Observation week } \\
\hline Pretreatment & $4.00 \mathrm{a}$ & $4.00 \mathrm{a}$ & $4.00 \mathrm{a}$ & $4.00 \mathrm{a}$ & $4.00 \mathrm{a}$ & $4.00 \mathrm{a}$ & $4.00 \mathrm{a}$ \\
\hline 1 & $3.36 \mathrm{~b}$ & $3.48 \mathrm{a}$ & $2.96 \mathrm{~b}$ & $3.96 \mathrm{a}$ & $4.00 \mathrm{a}$ & $3.88 \mathrm{a}$ & $3.80 \mathrm{a}$ \\
\hline 2 & $3.40 \mathrm{~b}$ & $3.60 \mathrm{a}$ & $3.00 \mathrm{~b}$ & $3.96 \mathrm{a}$ & $4.00 \mathrm{a}$ & $3.86 \mathrm{a}$ & $3.88 \mathrm{a}$ \\
\hline \multicolumn{8}{|c|}{ Year 2} \\
\hline \multicolumn{8}{|c|}{ Temp treatment $\left({ }^{\circ} \mathrm{C}\right)$} \\
\hline Control (22) & $3.90 \mathrm{ab}$ & $4.00 \mathrm{a}$ & $4.00 \mathrm{a}$ & $4.00 \mathrm{a}$ & $4.00 \mathrm{a}$ & $4.00 \mathrm{a}$ & $4.00 \mathrm{a}$ \\
\hline 35 & $3.98 \mathrm{a}$ & $4.00 \mathrm{a}$ & $4.00 \mathrm{a}$ & $4.00 \mathrm{a}$ & $4.00 \mathrm{a}$ & $4.00 \mathrm{a}$ & $4.00 \mathrm{a}$ \\
\hline 40 & $4.00 \mathrm{a}$ & $4.00 \mathrm{a}$ & $4.00 \mathrm{a}$ & $4.00 \mathrm{a}$ & $3.98 \mathrm{a}$ & $4.00 \mathrm{a}$ & $4.00 \mathrm{a}$ \\
\hline 45 & $4.00 \mathrm{a}$ & $3.66 \mathrm{~b}$ & $3.88 \mathrm{~b}$ & $3.96 \mathrm{a}$ & $4.00 \mathrm{a}$ & $4.00 \mathrm{a}$ & $3.78 \mathrm{a}$ \\
\hline 50 & $3.44 \mathrm{~b}$ & $3.08 \mathrm{c}$ & $3.96 \mathrm{ab}$ & $2.94 \mathrm{~b}$ & $3.88 \mathrm{~b}$ & $3.12 \mathrm{~b}$ & $2.92 \mathrm{~b}$ \\
\hline \multicolumn{8}{|c|}{ Observation week } \\
\hline Pretreatment & $4.00 \mathrm{a}$ & $4.00 \mathrm{a}$ & $4.00 \mathrm{a}$ & $4.00 \mathrm{a}$ & $4.00 \mathrm{a}$ & $4.00 \mathrm{a}$ & $4.00 \mathrm{a}$ \\
\hline 1 & $3.90 \mathrm{a}$ & $3.36 \mathrm{bc}$ & $3.88 \mathrm{~b}$ & $3.58 \mathrm{~b}$ & $3.96 \mathrm{a}$ & $3.76 \mathrm{a}$ & $3.66 \mathrm{a}$ \\
\hline 2 & $3.84 \mathrm{a}$ & $3.58 \mathrm{c}$ & $4.00 \mathrm{a}$ & $3.70 \mathrm{ab}$ & $3.98 \mathrm{a}$ & $3.78 \mathrm{a}$ & $3.60 \mathrm{a}$ \\
\hline
\end{tabular}

${ }^{\mathrm{z}}$ Rating is an average of 25 repetitions: $0=$ dead/completely brown leaves, $1=$ more than $60 \%$ brown with green still on leaves, $2=$ drooping/flagging leaves with a less than $50 \%$ brown or graying, $3=$ drooping/flagging leaves, but otherwise green leaves, $4=$ turgid, healthy, bright green leaves.

$\mathrm{y}\left(1.8 \times{ }^{\circ} \mathrm{C}\right)+32={ }^{\circ} \mathrm{F}$.

${ }^{\mathrm{x}}$ Values followed by different letters are significantly different within a cultivar, across treatment or week using Tukey's mean separation test at $P \leq 0.05$.

control potential root rot pathogen infection, several sanitation practices were implemented, including media drenches early in the production stage, as would be typical in a production system. Moreover, new, soilless substrate and pots were used in these experiments, and it is uncommon for new materials to contain pathogens (Daughtrey and Benson, 2005).

Other cultural considerations that may influence root ratings include watering, electrical conductivity (EC), and $\mathrm{pH}$. It is possible that if a plant stayed too wet or was too dry, root ratings could decrease. During our experiment dahlias were irrigated before leaves flagged and irrigation practices were as consistent as possible. $\mathrm{pH}$ and $\mathrm{EC}$ were monitored during the production cycle and our observations were found to be within the normal recommended range for dahlia production (data not shown). No salt accumulation (increased EC) that could burn the roots was observed. With the consistencies with watering, $\mathrm{EC}$, and $\mathrm{pH}$, and the unlikely possibility of pathogen influence, we can assume that the root rating decreases observed were in fact due to the treatments applied and how the individual cultivars reacted to them.

\section{Conclusions}

Some dahlia cultivars, when exposed to supraoptimal RZT, experience some level of root damage both initially and subsequently over time, can recover with maintaining potential market value. It has been hypothesized that supraoptimal RZTs may be a primary factor in dahlia decline. However, in our study, dahlia decline was not induced in any treatment for any cultivar with supraoptimal RZT exposure during our experiments. This suggests that a heat-damaged root zone may not be a primary factor that leads to this phenomenon.

It was insightful to learn that even with exposure to supraoptimal RZTs, and root damage, dahlia plants can recover. Based on our research, some cultivars are more adaptable to exposure to elevated RZTs and can rebound, whereas others may not. There is credible evidence that dahlia RZTs that reach above $40^{\circ} \mathrm{C}$ can cause root injury. The instances where dahlia root zones could encounter these temperatures for periods of time may include hot water left in a hose, extreme pot temperatures, or extreme greenhouse air temperatures. However, hot water left in a hose would not 


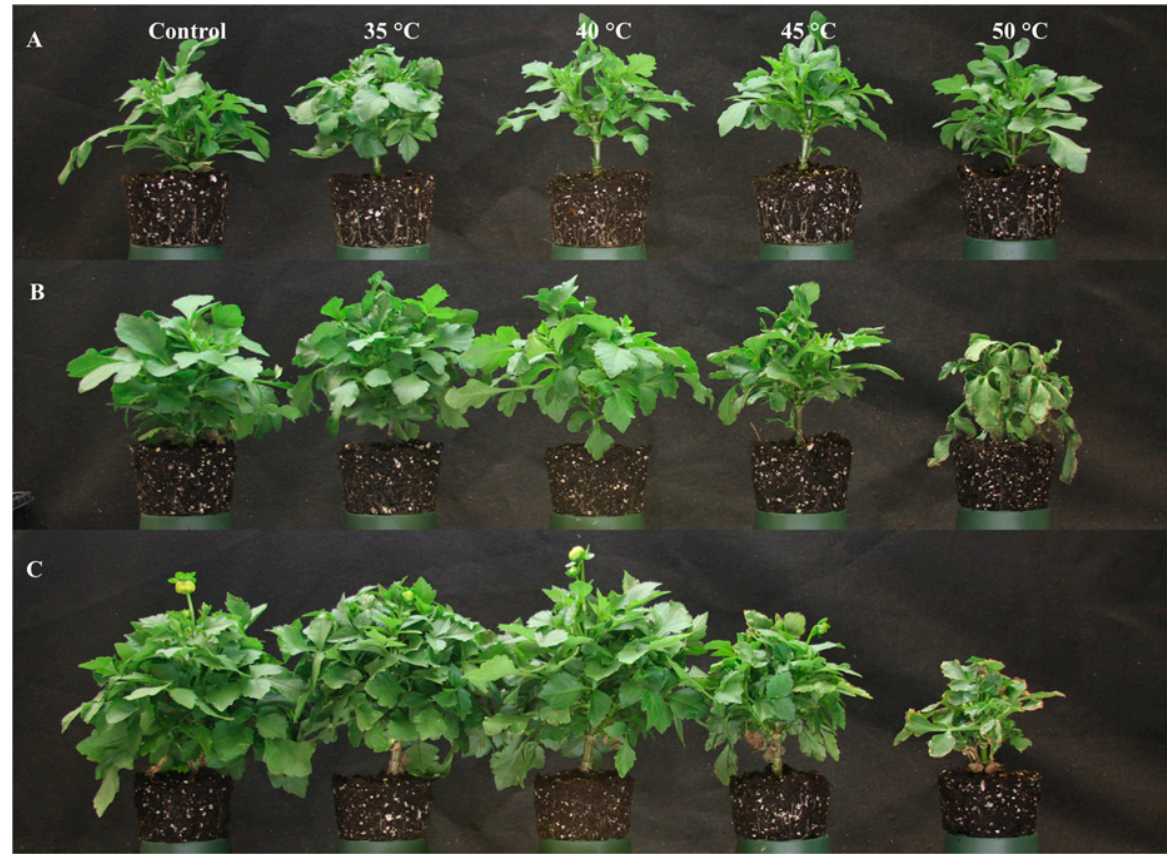

Fig. 4. Treatment comparisons for year 2 of 'Dalaya Shiva' dahlia. Representative plants from treatments are shown left to right in the photo; 22 (control), 35, 40, 45, 50 ${ }^{\circ} \mathrm{C}$ : (A) pretreatment observations, (B) 1 week posttreatment, and (C) 4 weeks posttreatment. Research was conducted in glass houses from 2 Feb. to 9 Apr. 2020 at Manhattan, $\mathrm{KS} ;\left(1.8 \times{ }^{\circ} \mathrm{C}\right)+32={ }^{\circ} \mathrm{F}$.

reliably explain entire crop losses, as the quantity of potential hot water would not be sufficient to kill a whole

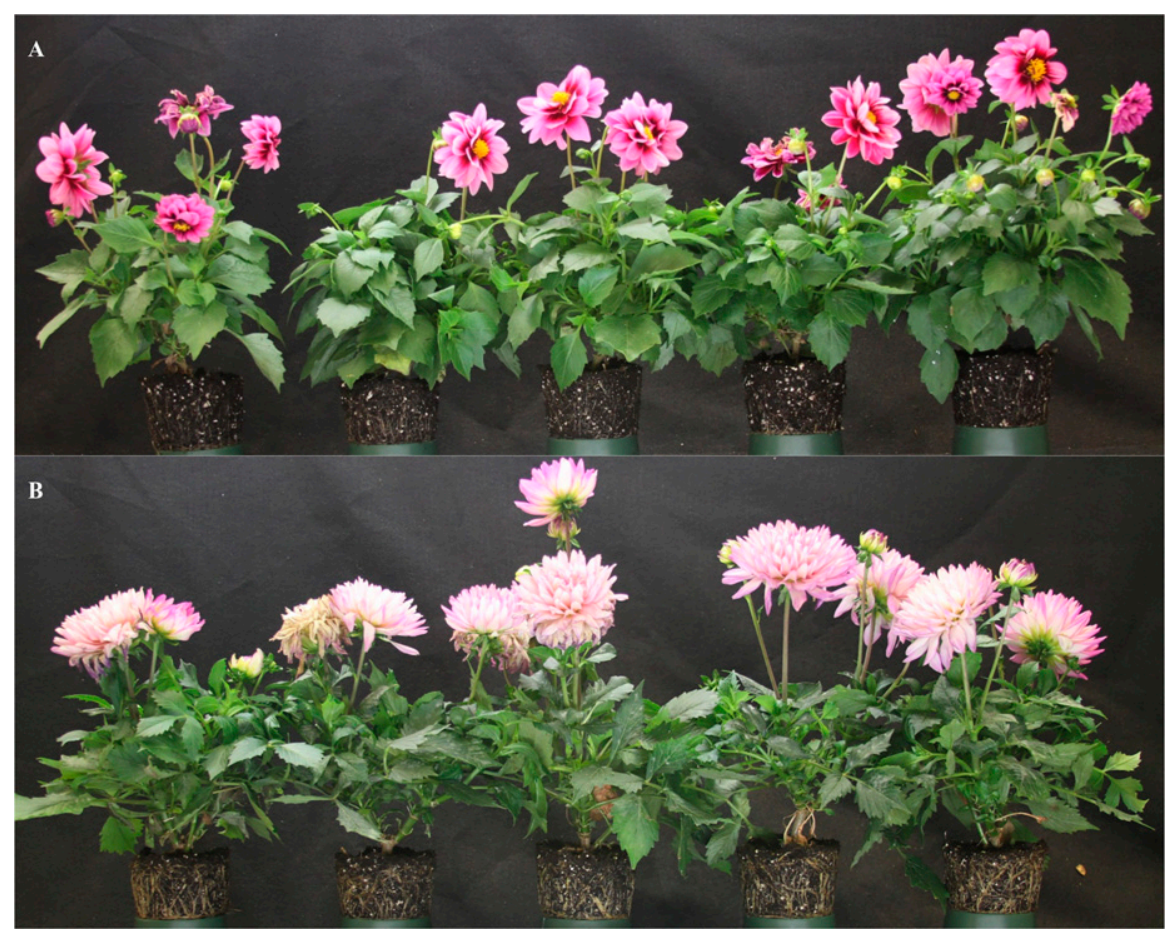

Fig. 5. Root rating differences 4 weeks posttreatment between two dahlia cultivars in year 2: (A) Dalaya Yogi control repetitions, and (B) XXL Veracruz $45^{\circ} \mathrm{C}\left(113.0^{\circ} \mathrm{F}\right)$ repetitions. Research was conducted in glass houses from $2 \mathrm{Feb}$. to 2 May 2020 at Manhattan, $\mathrm{KS} ;\left(1.8 \times{ }^{\circ} \mathrm{C}\right)+32={ }^{\circ} \mathrm{F}$. greenhouse would experience increased RZTs, based on solar radiation on the pot, is probably not likely, at least to the temperatures tested in these experiments. Pot and media surface temperatures were taken one day during our experiment; the highest average temperature was $35^{\circ} \mathrm{C}$ at 11:00 $\mathrm{Am}$ for pots on perimeter, south-facing side of the bench (data not shown). With this information, growers can make more educated decisions about their crops, especially if the crop endures elevated temperature exposure.

Dahlia decline appears to be a more complex combination of factors, including physiological or environmental causes or both. Supraoptimal RZT exposure does not appear to be one of the primary causes of dahlia decline, and future research is needed to investigate other plausible causal agents of the disorder.

\section{Literature cited}

Beckerman, J. 2011. Pythium root rot of herbaceous plants. Purdue Univ. Ext. Publ. BP-181-W.

Daughtrey, M.L. and M.D. Benson. 2005. Principles of plant health management for ornamental plants. Annu. Rev. Phytopathol. 43:141-169, doi: 10.1146/ annurev.phyto.43.040204.140007.

Dodd, I.C., J. He, C.G.N. Turnbull, S.K. Lee, and C. Critchley. 2000. The influence of supra-optimal root-zone temperatures on growth and stomatal conductance in Capsicum annuum L. J. Expt. Bot. 5l(343):239-248, doi: 10.1093/jexbot/ 51.343 .239

Dolce, J. 2020. Dahlias: Every gardener's delight. 7 July 2021. <https://gpnmag. com $/ 2020 / 01 /$ dahlias-every-gardenersdelight $/>$.

Du, Y.C. and S. Tachibana. 1994. Effect of supraoptimal root temperature on the growth, root respiration and sugar content of cucumber plants. Scientia Hort. 58(4):289-301, doi: 10.1016/0304-4238 (94)90099-X.

Hood, T.M. and H.A. Mills. 1994. Rootzone temperature affects nutrient uptake and growth of snapdragon. J. Plant Nutr. 17(2-3):279-291, doi: 10.1080/019041 69409364727.

Ingram, D.L., J.M. Ruter, and C.A. Martin. 2015. Review: Characterization and impact of supraoptimal root-zone temperatures in container-grown plants. HortScience 50(4): 530-539, doi: 10.21273/HORTSCI.50. 4.530 . 
Martin, C.A., D.L. Ingram, and T.A. Nell. 1989. Supraoptimal root-zone temperature alters growth and photosynthesis of holly and elm. J. Arboric. 15(11):272-276.

Martin, C.A., D.L. Ingram, and M.A. Jenks. 1991. Response of southern magnolia to supraoptimal root-zone temperatures. J. Therm. Biol. 16(5):281-285, doi: $10.1016 / 0306-4565(91) 90018-W$.

Moorman, G.W. and M.L. Daughtrey. 2002. Don't expect pythium root rot to always act the same. 7 July 2021. < https:// gpnmag.com/article/dont-expect-pythiumroot-rot-always-act-same $/>$.

Nambuthiri, S., A. Fulcher, A.K. Koeser, R. Geneve, and G. Niu. 2015. Moving toward sustainability with alternative containers for greenhouse and nursery crop production: A review and research update. HortTechnology 25(1):8-16, doi: 10.21273/HORT TECH.25.1.8.
National Garden Bureau. 2019. Year of the dahlia. I July 2021. <https://ngb. org/year-of-the-dahlia/ $>$.

Olberg, M.W. and R.G. Lopez. 2016. Growth and development of poinsettia (Euphorbia pulcherrima) finished under reduced air temperature and bench-top rootzone heating. Scientia Hort. 210(10):197204, doi: 10.1016/j.scienta.2016.07.017.

Pappu, H.R., S.D. Wyatt, and K.L. Druffel. 2005. Dahlia mosaic virus: Molecular detection and distribution in dahlia in the United States. HortScience 40(3): 697-699, doi: 10.21273/HORTSCI.40. 3.697 .

Sattelmacher, B., H. Marschner, and R. Kühne. 1990. Effects of the temperature of the rooting zone on the growth and development of roots of potato (Solanum tuberosum). Ann. Bot. 65(1):27-36, doi: 10.1093/oxfordjournals.aob.a087903.
Schoellhorn, R. 2015. Dahlia production tips for high quality greenhouse plants. 7 July 2021. <https://www.greenhouse grower.com/crops/dahlia-production-tipsfor-high-quality-greenhouse-plants $/>$.

Syngenta Flowers. 2019. Dahlia Sincerity culture guide. 6 July 2021. <https: / wwww. syngentaflowers-us.com/sites/g/files/zhg $721 / \mathrm{f} / \mathrm{media} / 2019 / 08 / 19 /$ culture vegetative_dahlia_sincerity__0.pdf $>$.

Wai, K.S. and S.E. Newman. 1992. Air root-zone temperatures influence growth and flowering of snapdragons. HortScience 27(7):796-798, doi: 10.21273/ HORTSCI.27.7.796.

Yeager, T.H., R.H. Harrison, and D.L. Ingram. 1991. 'Rotundifolia' holly growth and nitrogen accumulation influenced by supraoptimal root-zone temperatures. HortScience 26(11):1387-1388, doi: 10.21273/HORTSCI.26.11.1387. 